\title{
The frailty index is a predictor of cause-specific mortality independent of familial effects from midlife onwards: a large cohort study
}

Xia Li', Alexander Ploner ${ }^{1}$, Ida K. Karlsson ${ }^{2,1}$, Xingrong Liu', Patrik K. E. Magnusson ${ }^{1}$, Nancy L. Pedersen', Sara Hägg ${ }^{1}$ and Juulia Jylhävä ${ }^{*}$ (D)

\begin{abstract}
Background: Frailty index (FI) is a well-established predictor of all-cause mortality, but less is known for cause-specific mortality and whether familial effects influence the associations. Middle-aged individuals are also understudied for the association between $\mathrm{Fl}$ and mortality. Furthermore, the population mortality impact of frailty remains understudied.

Methods: We estimated the predictive value of FI for all-cause and cause-specific mortality, taking into account familial factors, and tested whether the associations are time-dependent. We also assessed the proportion of all-cause and cause-specific deaths that are attributable to increased levels of frailty. We analyzed 42,953 participants from the Screening Across the Lifespan Twin Study (aged 41-95 years at baseline) with up to 20 years' mortality follow-up. The $\mathrm{FI}$ was constructed using 44 health-related items. Deaths due to cardiovascular disease (CVD), respiratory-related causes, and cancer were considered in the cause-specific analysis. Generalized survival models were used in the analysis.

Results: Increased FI was associated with higher risks of all-cause, CVD, and respiratory-related mortality, with the corresponding hazard ratios of $1.28(1.24,1.32), 1.31(1.23,1.40)$, and $1.23(1.11,1.38)$ associated with a $10 \%$ increase in $\mathrm{Fl}$ in male single responders, and $1.21(1.18,1.25), 1.27(1.15,1.34)$, and $1.26(1.15,1.39)$ in female single responders. No significant associations were observed for cancer mortality. No attenuation of the mortality associations in unrelated individuals was observed when adjusting for familial effects in twin pairs. The associations were time-dependent with relatively greater effects observed in younger ages. Before the age of 80 , the proportions of deaths attributable to $\mathrm{FI}$ levels $>0.21$ were $18.4 \%$ of all-cause deaths, $25.4 \%$ of CVD deaths, and $20.4 \%$ of respiratory-related deaths in men and $19.2 \%$ of all-cause deaths, $27.8 \%$ of CVD deaths, and $28.5 \%$ of respiratory-related deaths in women. After the age of 80 , the attributable proportions decreased, most notably for all-cause and CVD mortality.

Conclusions: Increased Fl predicts higher risks of all-cause, CVD, and respiratory-related mortality independent of familial effects. Increased FI presents a relatively greater risk factor at midlife than in old age. Increased Fl has a significant population mortality impact that is greatest through midlife until the age of 80 .
\end{abstract}

Keywords: Frailty index, Mortality, Familial effect, Time-varying effect, Attributable fraction

\footnotetext{
* Correspondence: juulia.jylhava@ki.se

${ }^{1}$ The Department of Medical Epidemiology and Biostatistics, Karolinska

Institutet, Nobels väg 12A, 17165 Stockholm, Sweden

Full list of author information is available at the end of the article
}

(c) The Author(s). 2019 Open Access This article is distributed under the terms of the Creative Commons Attribution 4.0 International License (http://creativecommons.org/licenses/by/4.0/), which permits unrestricted use, distribution, and reproduction in any medium, provided you give appropriate credit to the original author(s) and the source, provide a link to the Creative Commons license, and indicate if changes were made. The Creative Commons Public Domain Dedication waiver (http://creativecommons.org/publicdomain/zero/1.0/) applies to the data made available in this article, unless otherwise stated. 


\section{Introduction}

Frailty is a major health concern associated with aging [1]. It is a state of multisystem physiological decline and inability to maintain homeostasis, gradually leading to an increased risk of multiple adverse outcomes, such as falls, hospitalizations, and death [1]. Frailty is also predictive of poor prognosis and post-operative complications in older surgical patients [2]. The two principal models to operationalize the frailty concept are the Rockwood frailty index (FI) and the Fried phenotypic model [3]. The FI defines the level of frailty as the ratio of the number of various health deficits present to the number of deficits considered whereas the FP classifies individuals as non-frail, pre-frail, or frail based on poor grip strength; slow walking speed; low physical activity, exhaustion; and unintentional weight loss. Being a continuous scale measure, the FI provides good sensitivity even at the lower end of the frailty continuum, which facilitates studies in younger individuals $[4,5]$.

The FI is associated with all-cause mortality in different populations, independent of other major risk factors [6]. The FI-mortality relationship is well-established, particularly among older individuals ( $>65$ years), but less is known for younger adults and for cause-specific mortality-aspects that may have implications for prevention. Both frailty and risk of death can be influenced by familial background, such as genetic and shared environmental factors, which may confound frailty-mortality associations [7]. However, the extent to which the predictive ability of the FI is affected by familial influences has not been studied. Sex-specific characteristics for the frailty-mortality association have also been suggested, but the matter remains inconclusive [8].

Despite the recognition of frailty as a public health concern [9], its direct population mortality impact has received less attention. One way to assess the public health relevance of frailty is to estimate the proportion of deaths that could be delayed if the levels of frailty were decreased in the population. Assessing the fraction of deaths that are attributable to increased frailty, considering both all-cause and cause-specific mortality as outcomes, would provide more precise information about public health relevance.

Consequently, this study aims to estimate the predictive value of the FI on all-cause and cause-specific mortality, separately for men and women. We assess the associations among both unrelated individuals and within twin pairs, to account for familial effects and to assess the degree to which frailty-mortality associations in the general population could be due to shared familial confounding. Secondly, we estimate FI-mortality associations in a time-dependent manner to elucidate the predictive value of FI from midlife and onwards. Lastly, we investigate the number of deaths that could be attributable to increased population levels of frailty by estimating generalized attributable fractions (GAFs) [10-12].

\section{Methods}

\section{Study population}

Data of the present study came from Screening Across the Lifespan Twin Study (SALT), which is part of the Swedish Twin Registry (STR) [13]. In 1998-2002, a total of 44,919 twins born between 1896 and 1958 were interviewed by a structured, computer-assisted telephone interview to collect information about illnesses and health, prescription and nonprescription medication use, occupation, education, and lifestyle behaviors. Twins were also asked to go to their local health care centers and provide blood for analyses of clinical chemistries and zygosity determination.

In the present study, we excluded participants who had more than $20 \%$ missing data across the 44 frailty items, and those for whom information on cause-specific mortality could not be retrieved. Eventually, 42,953 twin individuals aged from 41 to 95 years were included in the study, including 11,087 twin individuals whose partner did not respond, 11,548 opposite-sex dizygotic (DZ) twin individuals (5774 twin pairs), 11,812 same-sex DZ twin individuals (5906 twin pairs), and 8506 monozygotic (MZ) twin individuals (4253 twin pairs). Participants were further grouped into single responders, same-sex dizygotic (DZ) twin pairs, and monozygotic (MZ) twin pairs in the analyses, separately for men and women (Additional file 1: Table S1). In the sex-specific analyses, single responders included twins whose partner did not respond in SALT, twins from opposite-sex twin pairs, and one randomly selected member of each same-sex twin pair.

\section{Assessment of the FI}

The FI in SALT was constructed based on self-reported data using the Rockwood deficit accumulation model according to a standard procedure [4]. Briefly, the deficits to be included in the FI had to have $a \geq 1 \%$ prevalence and $\leq 10 \%$ missingness rate in the study population. Forty-four symptoms, signs, disabilities, and diseases covering a wide range of biological systems and associated with health status were considered in the FI. The items and scoring of the deficits are presented in Additional file 1: Table S2. Imputation was used to replace missing values in order to maximize the utilization of the data. Specific methods for the imputation and sensitivity analysis for the imputed data are presented in Additional file 1: Appendix S1.

An FI value for each individual was calculated as the number of deficits present divided by the total number of deficits $(n=44)$. For example, an individual having 8 deficits has an FI of $8 / 44=0.18$. To assess the validity of FI in SALT, we examined the distribution and associations with age. The FI was treated as a continuous variable with $10 \%$ increase per unit when modeling survival (hazard ratio, HR). 


\section{Assessment of mortality}

All-cause mortality data, including dates of deaths, were obtained from linkages of the STR to Swedish national registers through the personal identification number assigned to all residents. All-cause mortality data used in this study were updated on December 31, 2017, yielding up to 20 years of follow-up.

Cause-specific mortality data were obtained from the Cause of Death Register (CDR), with the latest update on December 31, 2014, yielding up to 17 years of follow-up. The CDR records include information about the underlying and contributory causes of death for all individuals registered as Swedish residents in the year of their death. Causes of death were recorded using the International Classification of Diseases (ICD) codes, with ICD-10 from 1997 and onwards. We considered cardiovascular disease (CVD; including stroke), respiratory-related causes, and cancer as the specific causes of death. The ICD codes included in each cause and the consensus classification used when more than one cause of death was recorded are presented in Additional file 1: Tables S3 and S4, respectively.

\section{Assessment of covariates}

Years of education, tobacco use status, body mass index (BMI), and history of diseases were assessed from the self-reported data in SALT. Tobacco status was categorized as non-user (reference category) or user if the person was currently smoking or using smokeless tobacco regularly or had previously smoked or used smokeless tobacco regularly. BMI was calculated as weight divided by height squared $\left(\mathrm{kg} / \mathrm{m}^{2}\right)$. Individuals were classified as having a history of CVD, chronic respiratory diseases, and/or cancer, if he/she reported any of the following conditions respectively: (1) angina pectoris, myocardial infarction, heart failure, stroke, high blood pressure, claudication, irregular cardiac rhythm/atrial fibrillation, circulation problems in limbs, or thrombosis, (2) asthma or chronic lung disease, (3) cancer, tumor disease, or leukemia.

\section{Statistical methods}

We used generalized survival models (GSMs) for the association between FI and mortality [14, 15]. Briefly, these are a generalization of flexible parametric survival models where the underlying baseline hazard is fitted as a smooth spline term, in our case via a natural cubic spline with 3 to 5 degrees of freedom for different outcomes and cohorts. We chose GSMs over conventional Cox regression for both computational and conceptual reasons-faster model fit for time-varying effects, easier estimation and extraction of time-varying effects and survival function from the fitted model, but also less restrictive assumptions when estimating attributable fractions/generalized impact fractions than for the Cox model [16]. Given the wide age range and long follow-up among participants, we used attained age as underlying time scale for all models to eliminate the possibility of non-proportional effects of FI that were driven by chronological age. Subjects in the cause-specific analyses were censored at the date when they died from other causes than the current outcome of interest, or at end of follow-up.

We first tested the association with survival assuming a time-constant effect among single responders, same-sex DZ twin pairs, and MZ twin pairs (see Additional file 1: Table S1 for grouping). Next, we tested if there were significant time-dependent effects for those causes of death that were significant in the time-constant model among single responders. To elucidate sex-specific characteristics of the associations, all analyses were performed separately for men and women. Years of education, tobacco use, and BMI were included as covariates in all models. For the cause-specific analyses, history of cancer, CVD, or chronic respiratory diseases at baseline were included as a covariate when the corresponding cause of death was the outcome of interest, see Additional file 1: Appendix S2, Eq.1, for the basic models for unrelated individuals.

For the same-sex DZ and MZ twin pairs, a betweenwithin decomposition along with a random effect from a gamma distribution was introduced to the GSM, which allow us to adjust for shared familial effects both due to shared patterns of exposure and confounders included in the model as well as general unmeasured similarity in survival patterns in twin pairs $[16,17]$. We have previously used a similar model in twin data for telomere length and survival [18]. The model is described in detail in Additional file 1: Appendix S2, Eq. 2.

For the subgroup of single responders, we also included interaction terms that allow the association between FI and survival to vary with age at assessment as well as time since assessment; details are presented in Additional file 1: Appendix S2, Eq. 3.

The generalized attributable fraction (GAF), in the literature also referred to as generalized impact fraction [12], was used to quantify the potential public health impact of FI on the survival outcome based on the time-constant survival models $[11,12,16]$. The GAF is an integrated predictive measure that takes into account both the distribution of a risk factor in the population as well as the strength of association between risk factor and outcome. Unlike the classical attributable fraction, it is also defined for a continuous exposure, eliminating the need to re-fit our models with dichotomized FI as exposure. In this study, we report the GAF function $[12,19]$, which shows the proportion of deaths that could potentially be prevented before a given age, or in other words, delayed beyond a certain age through a hypothetical intervention. In our approach, we assessed the mortality impact of a hypothetical intervention among 
those individuals who have an FI above a threshold of (a) 0.10 (i.e., the threshold for "least fit" [20]) and (b) 0.21 (i.e., the threshold for "frail" [20]). That is, the individuals with an FI above these thresholds would be (hypothetically) intervened to reduce their counterfactual FI values just below these thresholds ( 0.099 or 0.209 , respectively) to achieve the given reduction in mortality.

As our definition of FI includes items related to CVD, respiratory diseases, and cancer, we created three "reduced" FIs that were stripped of either CVD-related items (angina pectoris, myocardial infarction, heart failure, stroke, high blood pressure, claudication, irregular cardiac rhythm/atrial fibrillation, circulation problems in limbs, and thrombosis), respiratory-related items (chronic lung diseases and asthma), or cancer. These reduced FIs were then used as exposures in a sensitivity analysis for the corresponding cause-specific survival models. An additional sensitivity analysis using the original 44-item FI was performed for respiratory-related mortality by including only the 17,905 non-tobacco users in the analysis.

$P$ values reported are two-sided, and the statistical significance level was set at $p<0.05$. All analyses were conducted using Stata 15.1 and R 3.4.3. Statistical analyses involving GSMs were implemented using the rstpm2 package 1.4.0 [21].

\section{Results}

Characteristics of the study population are presented in Table 1. Of 42,953 individuals, $46.4 \%$ were men and $47.3 \%$ were complete twin pairs. At baseline, the median level of FI was 0.097 in men and 0.119 in women. FI distributions were skewed with a long right tail in all groups (total cohort, males, and females) (Additional file 1: Figure S1). The single responders tended to be older, had a lower education, and used tobacco products more frequently (in men only) than those from complete pairs.

During 20 years of follow-up for all-cause mortality and 17 years of follow-up for cause-specific mortality since 1998-2002, 12,222 (28.5\%) deaths were recorded, with 3270 (7.6\%), 1051 (2.4\%), and 3302 (7.7\%) deaths due to CVD, respiratory-related causes, and cancer, respectively. After controlling for education, tobacco use, BMI, the history of corresponding diseases, and familial effects in twins, increased FI significantly predicted higher risks of deaths due to all causes, CVD, and respiratory-related causes in time-constant models in both men and women (Fig. 1). The between effects from

Table 1 Characteristics of the study population

\begin{tabular}{|c|c|c|c|c|c|c|}
\hline & \multicolumn{3}{|l|}{ Men $(N=19,924)$} & \multicolumn{3}{|l|}{ Women $(N=23,029)$} \\
\hline & Single responders ${ }^{a}$ & DZ twins ${ }^{b}$ & MZ twins & Single responders ${ }^{a}$ & DZ twins ${ }^{b}$ & MZ twins \\
\hline Number of participants ${ }^{c}$ & 15,473 & 5266 & 3636 & 17,321 & 6546 & 4870 \\
\hline Age at baseline, mean (SD) & $58.7(10.6)$ & $57.4(9.6)$ & $56.9(9.2)$ & $59.6(11.2)$ & $58.5(10.3)$ & $57.8(10.2)$ \\
\hline BMI $\left(\mathrm{kg} / \mathrm{m}^{2}\right)$, mean (SD) & $25.5(3.1)$ & $25.6(3.1)$ & $25.5(3)$ & $24.6(3.7)$ & $24.5(3.7)$ & $24.4(3.8)$ \\
\hline Education (year), mean (SD) & $10.4(3.2)$ & $10.6(3.2)$ & $10.8(3.2)$ & $10.4(3.2)$ & $10.5(3.2)$ & $10.8(3.2)$ \\
\hline Tobacco products used, $\%$ & 64.3 & 63.1 & 59.5 & 53.9 & 54.3 & 52.5 \\
\hline $\mathrm{Fl}$, median (IQR) & $0.097(0.102)$ & $0.094(0.097)$ & $0.091(0.097)$ & $0.125(0.125)$ & $0.119(0.125)$ & $0.119(0.119)$ \\
\hline \multicolumn{7}{|c|}{ History of diseases at baseline, \% } \\
\hline CVD & 35.5 & 34.0 & 31.5 & 37.4 & 35.6 & 34.8 \\
\hline Respiratory disease & 9.6 & 8.2 & 9.6 & 11.9 & 10.8 & 11.7 \\
\hline Cancer & 4.7 & 4.2 & 4.7 & 9.1 & 8.5 & 9.3 \\
\hline \multicolumn{7}{|l|}{ Time to follow-up, median (IQR) } \\
\hline All-cause mortality & $16.7(2.8)$ & $16.9(2.4)$ & $16.9(2.3)$ & $16.9(2.6)$ & $17.0(2.5)$ & $17.1(2.4)$ \\
\hline Cause-specific mortality & $14.0(2.5)$ & $14.1(2.3)$ & $14.1(2.3)$ & $14.2(2.5)$ & $14.3(2.2)$ & $14.3(2.3)$ \\
\hline \multicolumn{7}{|l|}{ Death during the follow-up, \% } \\
\hline All causes & 31.3 & 26.0 & 23.4 & 28.3 & 25.0 & 22.1 \\
\hline CVD & 8.9 & 7.0 & 5.7 & 7.4 & 6.1 & 4.8 \\
\hline Respiratory-related causes & 2.7 & 2.1 & 1.9 & 2.4 & 2.2 & 1.7 \\
\hline Cancer & 9.0 & 7.7 & 7.5 & 7.0 & 6.7 & 5.9 \\
\hline
\end{tabular}

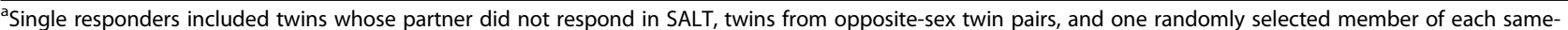
sex twin pair

${ }^{\mathrm{b}} \mathrm{DZ}$ twins refer to the same-sex DZ twins

cNumber of participants refers to the number of single responders or the number of individuals in DZ/MZ twin pairs

${ }^{\mathrm{d}}$ Participants who used tobacco products include current smokers, ex-smokers, and snuff users at baseline 


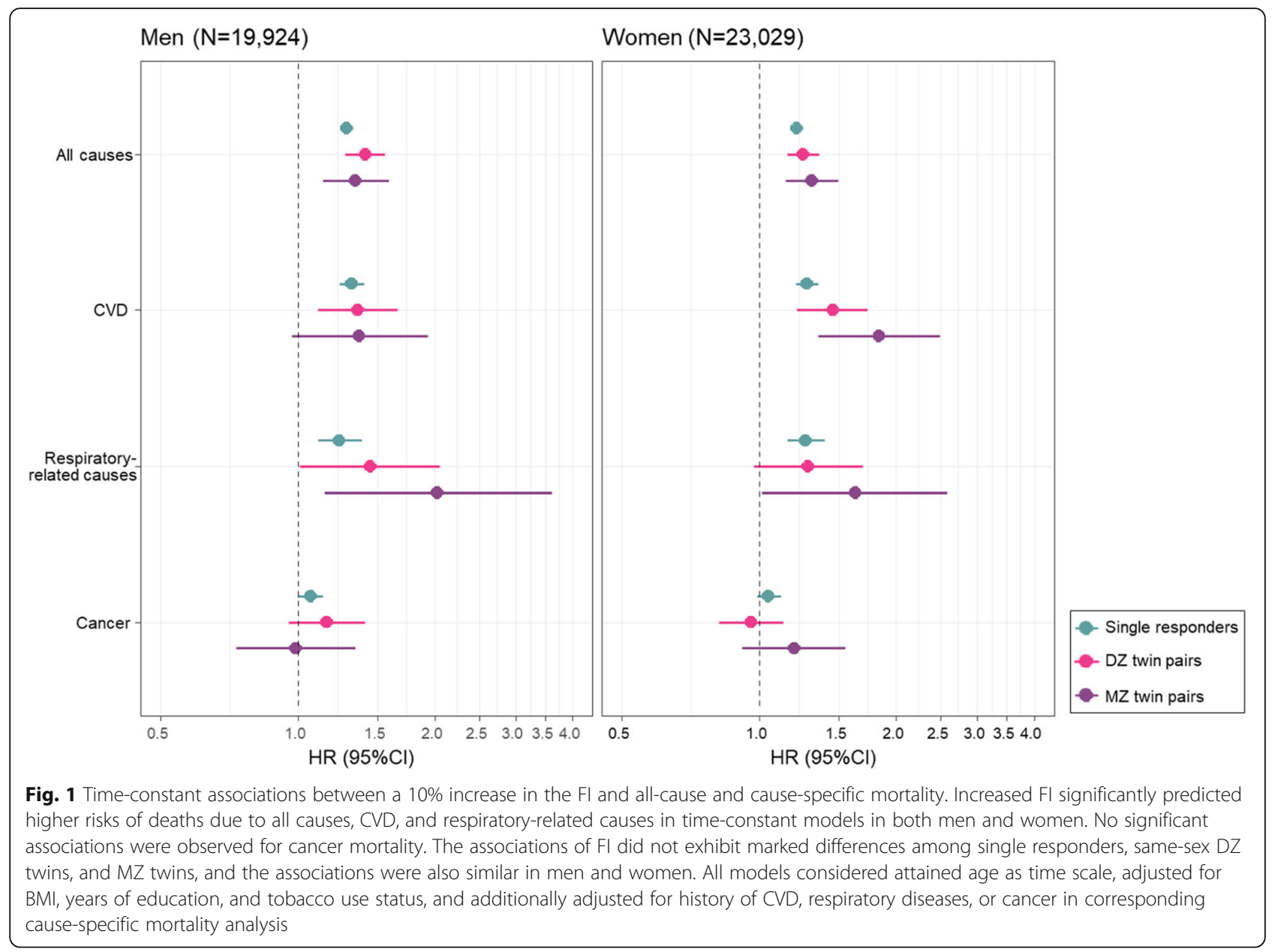

these models for twin pairs showed no significant associations, except for CVD mortality in MZ women (Additional file 1: Table S5). The corresponding hazard ratios (HRs) associated with a $10 \%$ increase in the FI were 1.28 (1.24, 1.32), 1.31 (1.23, 1.40), and $1.23(1.11,1.38)$ in male single responders and $1.21(1.18,1.25), 1.27$ (1.15, $1.34)$, and $1.26(1.15,1.39)$ in female single responders (Additional file 1: Table S5). No significant associations were observed for cancer mortality (Fig. 1). The associations between FI and mortality did not exhibit marked differences among single responders, same-sex DZ twins, and $\mathrm{MZ}$ twins and were also similar in men and women (Fig. 1). Additionally, we found time-dependent effects of FI for all-cause, CVD, and respiratory-related mortality in both men and women, with relatively greater HRs at younger ages and effect sizes decreasing with increasing age at FI assessment for all the causes (Fig. 2, single responders only), with a $1-2 \%$ decrease in strength of the association per extra year of age at assessment (Additional file 1: Table S6; single responders only).

Sensitivity analyses demonstrated that the association of FI and cause-specific mortality did not change appreciably when FI was stripped of item(s) related to the cause of death under examination, or when tobacco users were excluded from the study population (Additional file 1: Table S7).

The GAF estimates across the whole age range are presented in Fig. 3, and the GAFs (95\%CIs) before the age of 80 years are also provided in a numeric format in Additional file 1: Table S8. The GAFs demonstrated a decreasing trend towards the oldest ages and were relatively constant before the age of 80 . In men, the GAFs (95\%CI) revealed that $18.4 \%(16.1-20.7 \%)$ of all-cause deaths, $25.4 \%$ (20.1-30.8\%) of CVD deaths, and $20.4 \%$ (10.4-30.5\%) of respiratory-related deaths could be delayed beyond age 80 if the FI levels $>0.21$ were reduced in the population. In women, the corresponding GAFs were $19.2 \%(16.6-21.9 \%)$ for all-cause deaths, $27.8 \%$ (22-33.5\%) for CVD deaths, and 28.5\% (18.4-38.7\%) for respiratory-related deaths (Additional file 1: Table S8).

\section{Discussion}

In this study, we constructed the FI based on the Rockwood cumulative deficit model in a Swedish twin 


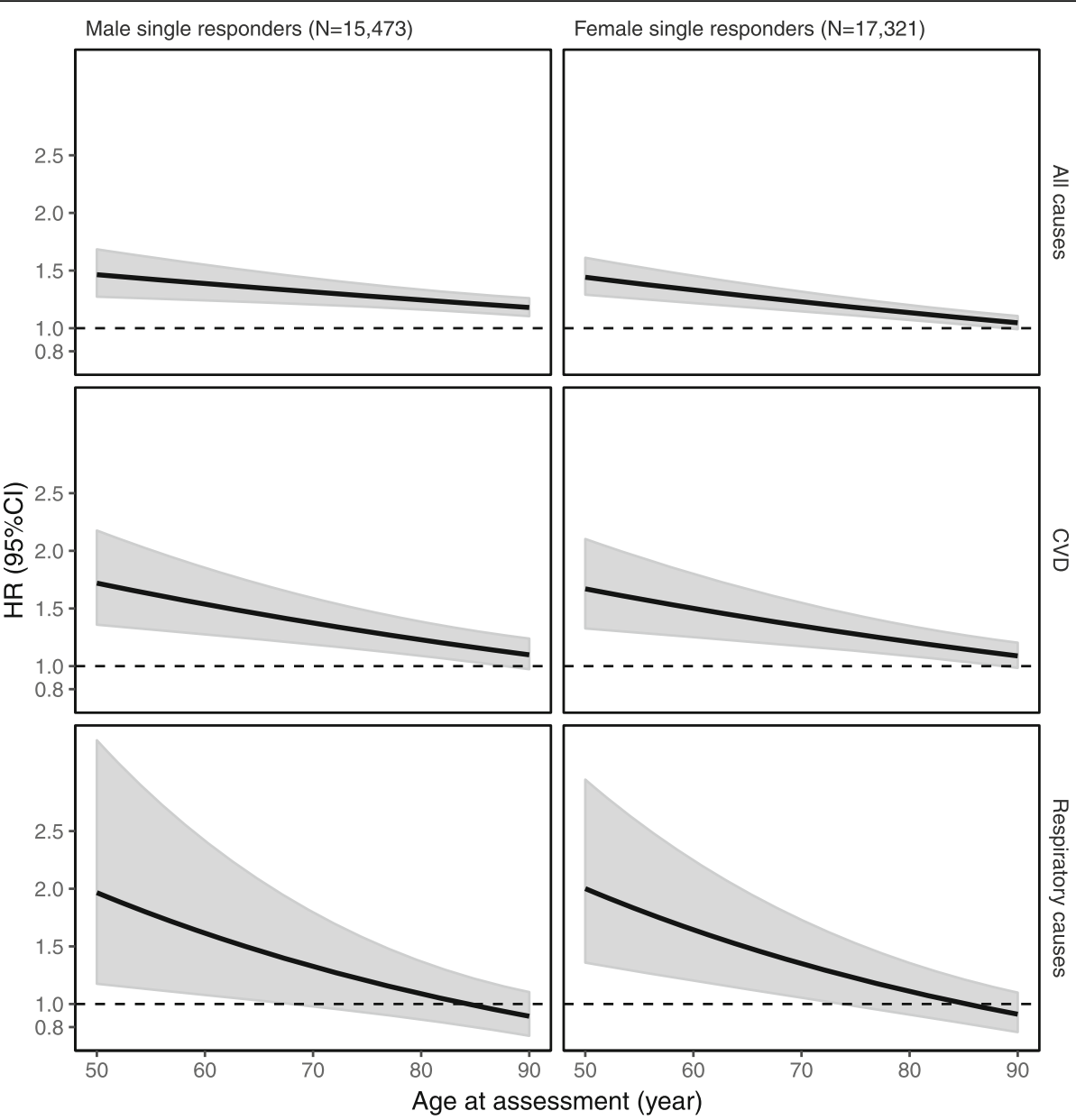

Fig. 2 Time-dependent associations between a 10\% increase in the Fl and mortality in single responders. Time-dependent effects of Fl for all-cause, CVD, and respiratory-related mortality in both men and women were observed, with relatively greater HRs at younger ages and effect sizes decreasing with increasing age at Fl assessment for all the causes. All models were adjusted for BMI, years of education, and tobacco use status, and additionally adjusted for history of CVD or respiratory diseases in corresponding cause-specific mortality analysis, and were fitted as a function of age at FI assessment

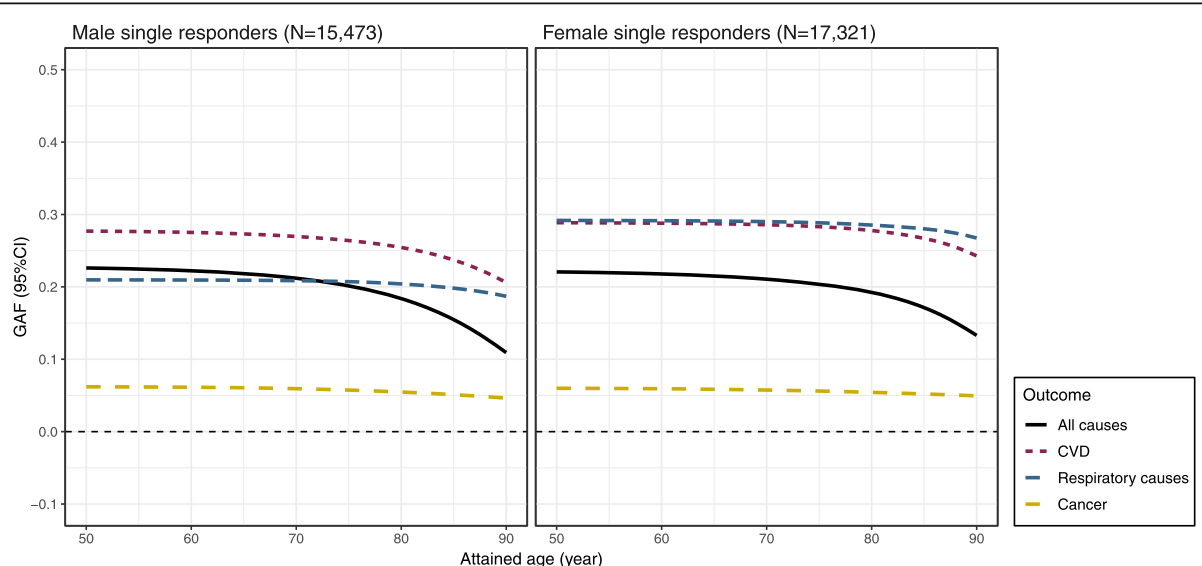

Fig. 3 Generalized attributable fraction (GAF) functions for all-cause and cause-specific mortality in single responders. The GAF represents the proportion of deaths that could be prevented before a given age (i.e., delayed beyond that age) if Fl levels greater than 0.21 were reduced to the level of 0.209 in the population. The estimated GAFs demonstrated that theoretical reduction of the FI levels below 0.20 would potentially delay 20-30\% of all-cause, CVD, and respiratory-related deaths in men and women beyond the age of 80 years. All models were adjusted for BMI, years of education, and tobacco use status, and additionally adjusted for history of CVD, or respiratory diseases in corresponding cause-specific mortality analysis, and were fitted as a function of age at $\mathrm{Fl}$ assessment 
population and assessed its predictive value on all-cause and cause-specific mortality. During the 20-year follow-up, an increase in FI conferred a significant risk for all-cause, CVD, and respiratory-related mortality, in both men and women. Relatively greater HRs were observed at younger ages; the relative risk conferred by a $10 \%$ increase in the FI was greatest at the attained age of 50 after which it decreased by $1-2 \%$ per year. The associations were persistent when adjusted for tobacco use, education, and BMI. When adjusting for familial effects in twin pairs, we found no attenuation of the associations between FI and mortality in comparison to those observed in single responders. In fact, even an increasing trend in the strength for FI and respiratory-related mortality in men and for FI and CVD mortality in women was found when the familial effects were accounted for. No associations with cancer mortality were however found. The estimated GAFs demonstrated that a (theoretical) reduction of the FI levels below 0.21 could prevent $18.4-28.5 \%$ of all-cause, CVD, and respiratory-related deaths in men and women before the age of 80 years. In line with the relatively greater mortality risks observed in younger ages, the GAFs were likewise greater at and across midlife and younger old ages, and decreased towards the oldest ages.

As frailty is considered to be a manifestation of aging, FI in relation to mortality has been mostly investigated in older populations $[6,22]$. Several studies with wide age spectra have nevertheless suggested that a negative effect of increased FI on mortality could also be observed in younger adults [20, 23-25]. In fact, previous findings by others [26] and us [23, 25] have suggested that the frailty-mortality association is relatively stronger among the young than the old. In the present study, we modeled the effect in a time-dependent manner and found that for all causes, higher FI was associated with a greater relative risk of mortality at younger ages and the associations attenuated towards the oldest ages. Such accumulating evidence suggests that a shift towards more research into frailty in younger populations is warranted and younger individuals should also be considered for frailty screening.

Although a wealth of evidence exists to demonstrate that a higher level of frailty is predictive of all-cause mortality, previous studies have mostly been conducted in unrelated individuals $[6,21]$. The associations may thus be confounded by familial factors, such as genetics and early life environment, affecting the risk of both frailty and mortality. We tested this by modeling the effects in MZ and DZ twin pairs, and found no evidence that the associations were attenuated compared to the results obtained in single responders, and the between models in twins suggested overall very little to no contribution of familiar factors to the associations. In other words, the FI is at least as useful a predictor of mortality after adjustment for shared family history in twins as in a general, unrelated population, suggesting that FI captures the individual association between frailty at time of assessment and risk of mortality with little or no confounding from shared familial lifestyle or genetic factors.

Frailty presents a sex-specific pattern that is similar to the male-female health-survival paradox in which women experience higher rates of disability and comorbidity yet still outlive men [27]. Women have higher levels of frailty throughout the age range, but men are more vulnerable to death at any given level of frailty $[4,8]$. However, whether frailty is a stronger risk factor for men than for women is currently unclear and different studies have reported contrasting results [28-32]. We tested this hypothesis by assessing the associations with both all-cause and causespecific mortality in men and women separately. Overall, the HRs were similar in men and women, even when adjusted for familial effects in twins. The time-dependent effects also demonstrated similar patterns in men and women, with relatively greater HRs observed at midlife and younger old ages and an attenuating trend towards the oldest ages.

Other studies that used the Fried phenotypic model instead of FI to measure frailty have also demonstrated that increased frailty is predictive of CVD mortality: Crow et al. reported an association that was adjusted for sex in an analysis including men and women [33], and Veronese et al. analyzed men and women separately and demonstrated that the association was stronger in women [34]. However, as analyses into cause-specific mortality and frailty are currently limited, more research is warranted to establish the relationship between frailty and cause-specific mortality. Regarding cancer mortality, a study in older breast cancer patients has shown that frailty is predictive of both all-cause and breast cancer mortality [35], and a systematic review has concluded that frailty is an independent predictor of all-cause mortality in older patients with various cancers [36]. Nevertheless, in the light of our finding that no significant association was observed between the FI and cancer mortality, it appears that the level of frailty is not predictive of cancer deaths in the general population of middle-aged and older individuals, most of whom do not have (or have had) cancer at the time of the FI assessment. Our previous results in a smaller STR sample have also shown that the suggestive association found between the FI and cancer mortality is attenuated after adjusting for present cancer diagnosis [23], indicating that the level of frailty might be a valid predictor of cancer mortality only among those who already have cancer.

Due to its association with various adverse outcomes, frailty presents a public health concern. The risk is not restricted to the highest end of the frailty continuum; 
lower levels of frailty, such as the pre-frailty state measured using the FP, are also predictive of mortality [3]. Therefore, we assessed the proportion of deaths that could be avoided by decreasing the levels of frailty using the cut-offs for "least fit" and "frail" according to Rockwood et al. [20]. We found that before the age of 80 , these proportions ranged from approximately 20 to $30 \%$ for all the causes, in both men and women, and the GAFs for the threshold of "least fit" were on average only $2 \%$ greater than those for "frail". Hence, the GAFs not only highlight a great potential for preventions aimed at reducing mortality by reducing the levels of FI, but also that most of the benefit from such an intervention would come from reducing the FI levels greater than 0.21 . Our GAF estimates for all-cause mortality are higher than previously reported in a systematic review that estimated the population risk of all-cause mortality attributable to frailty to be $3-5 \%$. The systematic review used a higher cut-off, corresponding to the frailty state, and the individuals included were older than 65 years [22]. These differences may account at least in part for the differing results. In fact, the GAFs in our study showed a decreasing trend towards the very old ages, indicating that higher frailty bears a relatively greater population risk of mortality at midlife and younger old ages. A recommendation to screen all individuals at 70 years and older for frailty at health care facilities has been put forward [37]. However, pertaining to mortality risk stratification, our results would suggest a potential benefit of considering also middle-aged individuals for frailty screening.

The present study has several strengths. First, we had a large population of twins that allowed us to control for unmeasured familial effects. Second, contrary to previous research mainly focusing on FI among older individuals (> 65 years), our cohort also included younger adults and had an age range from 40 to 95 years, which allowed us to model the association between frailty and mortality as a continuous function of age at assessment. Third, we had a very long follow-up, up to 20 years for all-cause mortality and up to 17 years for cause-specific mortality, which enables us to draw conclusions on the long-term predictive ability of the FI. Indeed, the associations in this study demonstrate that the FI is predictive of mortality in a long-term follow-up and align with a previous study by our group, in which we demonstrated that the FI is predictive of all-cause mortality for up to 30 years [23].

The present study also has some limitations. Frailty was measured using only one scale, the FI, and was based on self-reported data. Another limitation is that many of the included FI items were diseases or symptoms, making medical conditions having a relatively large weight in our FI. As our FI contained items of cancer, CVD, and chronic respiratory diseases, in the cause-specific analysis, we adjusted each of the models for the history of the given disease that was the cause of death in that analysis. For each of the cause-specific mortality analyses, we additionally created a modified FI that was stripped of the item(s) related to the cause of death under examination. Lastly, we repeated the analysis of respiratory-related mortality in non-tobacco users. All results remained unchanged in these sensitivity analyses, suggesting that the FI is a robust marker for predicting the risk of death due to $\mathrm{CVD}$ and respiratory-related causes, even in the presence of these diseases.

\section{Conclusions}

An increase in FI predicted higher risks of all-cause, CVD, and respiratory-related mortality, independent of familial effects, and exhibited time-dependent associations suggesting that a higher level of frailty is a relatively greater risk factor for middle-aged than for older individuals. Our results also highlight the significant population mortality impact of increased FI levels, especially above the threshold commonly considered to demarcate a "frail" state.

\section{Additional file}

\begin{abstract}
Additional file 1: Table S1. Grouping of the study population. Table S2. The 44 frailty items and the coding rules. Table S3. ICD codes used to classify the cause-specific mortality. Table S4. Consensus classification for the cause-specific mortality when more than one cause of death was recorded. Table S5. Time-constant associations between 10\% increase in the FI and all-cause and cause-specific mortality. Table S6. Assessment of time-dependent associations between FI and mortality in single responders. Table S7. Sensitivity analyses on the associations between $10 \% \mathrm{Fl}$ increase on all-cause and cause-specific mortality in single responders. Table S8. Generalized attributable fractions $(\%, 95 \% \mathrm{Cl})$ for all-cause and cause-specific mortality in single responders at the attained age of 80 . Table S9. Generalized attributable fractions (\%, 95\%Cl) for allcause and cause-specific mortality in twins at the attained age of 80 . Figure S1. Distribution of the frailty index in our sample. Appendix S1 Imputation of missing values. Appendix S2 Specification of generalized survival models (GSM) fitted to the data. (DOCX $66 \mathrm{~kb}$ )
\end{abstract}

\section{Abbreviations}

BMI: Body mass index; CDR: Cause of death register; CVD: Cardiovascular disease; DZ twins: Dizygotic twins; FI: Frailty index; GAF: Generalized attributable fractions; GSM: Generalized survival model; HR (95\%Cl): Hazard ratio (95\% confidence interval); HR: Hazard ratio; ICD: International Classification of Diseases; MZ twins: Monozygotic twins; SALT: Screening Across the Lifespan Twin Study; STR: Swedish Twin Registry

\section{Acknowledgements}

Not applicable.

\section{Funding}

This study was supported by NIH grants AG08724, the Swedish Research Council (2017-00641, 2015-03255, 2018-02077), FORTE (2013-2292), the Loo \& Hans Osterman Foundation, the Foundation for Geriatric Diseases, the Magnus Bergwall Foundation, the Strategic Research Program in Epidemiology at Karolinska Institutet (SH, J), and the China Scholarship Council.

\section{Availability of data and materials}

Data used in the current study are not publicly available due to ethical reasons. However, data are available upon request from the Swedish Twin Registry for researchers who meet the criteria for access to confidential data. 
Data from the SALT study are available from the Swedish Twin Registry steering committee (http://ki.se/en/research/the-swedish-twin-registry-1; contact: Patrik Magnusson' Patrik.Magnusson@ki.se, or Barbro Sandin, tvillingregistret@ki.se).

\section{Authors' contributions}

$X L i, A P, X L i u, N L P, S H$, and JJ contributed to the design of the present study and data analysis. $\mathrm{XLi}, \mathrm{AP}$, and JJ wrote the manuscript. AP was responsible for designing and supervising the statistical analysis. IKK contributed to the acquisition of the mortality data. PKEM and NLP are leaders of the SALT study and responsible for cohort recruitment, data collection, and funding. All authors contributed to interpretation of the results, and read and approved the final manuscript.

\section{Ethics approval and consent to participate}

Data collection in SALT was approved by the Research Ethics Committee at Karolinska Institutet (Dnr 97-051 and 00-132) and the current study by the Regional Ethics Board in Stockholm (Dnr 2016/1888-31/1). Informed consent was obtained from all participants. Participants were informed in writing prior to the computer-assisted telephone interview, with information that their participation was voluntary and that they could interrupt their participation at any time. At the end of the interview, participants were asked whether they could be contacted again for complementary questions and whether the Twin Registry could contact their physician to obtain medical records. After the interview, a letter was sent out to each participant confirming that they had been informed, had participated, and whether they agreed to recontact and/or contact with their physician.

\section{Consent for publication}

Not applicable.

\section{Competing interests}

The authors declare that they have no competing interests.

\section{Publisher's Note}

Springer Nature remains neutral with regard to jurisdictional claims in published maps and institutional affiliations.

\section{Author details}

${ }^{1}$ The Department of Medical Epidemiology and Biostatistics, Karolinska Institutet, Nobels väg 12A, 17165 Stockholm, Sweden. ${ }^{2}$ Institute of Gerontology and Aging Research Network - Jönköping (ARN-J), School of Health and Welfare, Jönköping University, Jönköping, Sweden.

\section{Received: 7 December 2018 Accepted: 29 April 2019}

\section{Published online: 15 May 2019}

\section{References}

1. Clegg A, Young J, lliffe S, Rikkert MO, Rockwood K. Frailty in elderly people. Lancet. 2013;381(9868):752-62.

2. Lin HS, Watts JN, Peel NM, Hubbard RE. Frailty and post-operative outcomes in older surgical patients: a systematic review. BMC Geriatr. 2016;16(1):157.

3. Fried LP, Tangen CM, Walston J, Newman AB, Hirsch C, Gottdiener J, Seeman T, Tracy R, Kop WJ, Burke G, et al. Frailty in older adults: evidence for a phenotype. J Gerontol A Biol Sci Med Sci. 2001;56(3):M146-56.

4. Searle SD, Mitnitski A, Gahbauer EA, Gill TM, Rockwood K. A standard procedure for creating a frailty index. BMC Geriatr. 2008;8:24.

5. Rockwood K, Mitnitski A. Frailty defined by deficit accumulation and geriatric medicine defined by frailty. Clin Geriatr Med. 2011;27(1):17-26.

6. Kojima G, lliffe S, Walters K. Frailty index as a predictor of mortality: a systematic review and meta-analysis. Age Ageing. 2018;47(2):193-200.

7. Young AC, Glaser K, Spector TD, Steves CJ. The identification of hereditary and environmental determinants of frailty in a cohort of UK twins. Twin Res Hum Genet. 2016;19(6):600-9.

8. Gordon EH, Peel NM, Samanta M, Theou O, Howlett SE, Hubbard RE. Sex differences in frailty: a systematic review and meta-analysis. Exp Gerontol. 2017:89:30-40.

9. Mitnitski A, Collerton J, Martin-Ruiz C, Jagger C, von Zglinicki T, Rockwood K, Kirkwood TB. Age-related frailty and its association with biological markers of ageing. BMC Med. 2015;13:161.
10. Chen YQ, Hu C, Wang Y. Attributable risk function in the proportional hazards model for censored time-to-event. Biostatistics. 2006;7(4):515-29.

11. Sjölander A, Vansteelandt S. Doubly robust estimation of attributable fractions in survival analysis. Stat Methods Med Res. 2017;26(2):948-69.

12. Taguri M, Matsuyama Y, Ohashi Y, Harada A, Ueshima H. Doubly robust estimation of the generalized impact fraction. Biostatistics. 2012;13(3):455-67.

13. Magnusson PK, Almqvist C, Rahman I, Ganna A, Viktorin A, Walum H, Halldner L, Lundström S, Ullén F, Långström N, et al. The Swedish Twin Registry: establishment of a biobank and other recent developments. Twin Res Hum Genet. 2013;16(1):317-29.

14. Liu XR, Pawitan Y, Clements M. Parametric and penalized generalized survival models. Stat Methods Med Res. 2018;27(5):1531-46.

15. Liu XR, Pawitan Y, Clements MS. Generalized survival models for correlated time-to-event data. Stat Med. 2017:36(29):4743-62.

16. Dahlqwist $E$, Pawitan $Y$, Sjölander $A$. Regression standardization and attributable fraction estimation with between-within frailty models for clustered survival data. Stat Methods Med Res. 2017. https://doi.org/10.1177/ 0962280217727558

17. Sjölander A, Lichtenstein P, Larsson H, Pawitan Y. Between-within models for survival analysis. Stat Med. 2013;32(18):3067-76.

18. Zhan Y, Liu XR, Reynolds CA, Pedersen NL, Hägg S, Clements MS. Leukocyte telomere length and all-cause mortality: a between-within twin study with time dependent effects using generalized survival models. Am J Epidemiol. 2018.

19. Chen L, Lin DY, Zeng D. Attributable fraction functions for censored event times. Biometrika. 2010;97(3):713-26.

20. Rockwood K, Song X, Mitnitski A. Changes in relative fitness and frailty across the adult lifespan: evidence from the Canadian National Population Health Survey. CMAJ. 2011;183(8):E487-94.

21. Mark Clements and Xing-Rong Liu: rstpm2: generalized survival models. $\mathrm{R}$ package version 1.4.1. 2018. http://github.com/mclements/rstpm2.

22. Shamliyan T, Talley KM, Ramakrishnan R, Kane RL. Association of frailty with survival: a systematic literature review. Ageing Res Rev. 2013;12(2):719-36.

23. Jiang $M$, Foebel AD, Kuja-Halkola R, Karlsson I, Pedersen NL, Hägg S, Jylhävä J. Frailty index as a predictor of all-cause and cause-specific mortality in a Swedish population-based cohort. Aging (Albany NY). 2017:9(12):2629-46.

24. Saum KU, Dieffenbach AK, Müller H, Holleczek B, Hauer K, Brenner H. Frailty prevalence and 10-year survival in community-dwelling older adults: results from the ESTHER cohort study. Eur J Epidemiol. 2014;29(3):171-9.

25. Williams DM, Jylhävä J, Pedersen NL, Hägg S. A frailty index for UK Biobank participants; 2017

26. Kulminski AM, Ukraintseva SV, Culminskaya IV, Arbeev KG, Land KC, Akushevich L, Yashin Al. Cumulative deficits and physiological indices as predictors of mortality and long life. J Gerontol A Biol. 2008;63(10):1053-9.

27. Oksuzyan A, Juel K, Vaupel JW, Christensen K. Men: good health and high mortality. Sex differences in health and aging. Aging Clin Exp Res. 2008; 20(2):91-102.

28. Yang F, Gu DN. Predictability of frailty index and its components on mortality in older adults in China. BMC Geriatr. 2016;16:145.

29. Bartley MM, Geda YE, Christianson TJ, Pankratz VS, Roberts RO, Petersen RC. Frailty and mortality outcomes in cognitively normal older people: sex differences in a population-based study. J Am Geriatr Soc. 2016;64(1):132-7.

30. Song X, Mitnitski A, Rockwood K. Age-related deficit accumulation and the risk of late-life dementia. Alzheimers Res Ther. 2014;6(5-8):54.

31. Shi J, Song X, Yu P, Tang Z, Mitnitski A, Fang X, Rockwood K. Analysis of frailty and survival from late middle age in the Beijing longitudinal study of aging. BMC Geriatr. 2011;11:17

32. García-González JJ, García-Peña C, Franco-Marina F, Gutiérrez-Robledo LM. A frailty index to predict the mortality risk in a population of senior Mexican adults. BMC Geriatr. 2009;9:47.

33. Crow RS, Lohman MC, Titus AJ, Bruce ML, Mackenzie TA, Bartels SJ, Batsis JA. Mortality risk along the frailty Spectrum: data from the National Health and Nutrition Examination Survey 1999 to 2004. J Am Geriatr Soc. 2018;66(3):496-502.

34. Veronese N, Sigeirsdottir K, Eiriksdottir G, Marques EA, Chalhoub D, Phillips $C L$, Launer LJ, Maggi S, Gudnason V, Harris TB. Frailty and risk of cardiovascular diseases in older persons: the age, gene/environment susceptibility-Reykjavik study. Rejuvenation Res. 2017;20(6):517-24. 
35. Mandelblatt JS, Cai L, Luta G, Kimmick G, Clapp J, Isaacs C, Pitcher B, Barry W, Winer E, Sugarman S, et al. Frailty and long-term mortality of older breast cancer patients: CALGB 369901 (Alliance). Breast Cancer Res Treat. 2017;164(1):107-17.

36. Handforth C, Clegg A, Young C, Simpkins S, Seymour MT, Selby PJ, Young J. The prevalence and outcomes of frailty in older cancer patients: a systematic review. Ann Oncol. 2015;26(6):1091-101.

37. Morley JE, Vellas B, van Kan GA, Anker SD, Bauer JM, Bernabei R, Cesari M, Chumlea WC, Doehner W, Evans J, et al. Frailty consensus: a call to action. J Am Med Dir Assoc. 2013;14(6):392-7.

Ready to submit your research? Choose BMC and benefit from:

- fast, convenient online submission

- thorough peer review by experienced researchers in your field

- rapid publication on acceptance

- support for research data, including large and complex data types

- gold Open Access which fosters wider collaboration and increased citations

- maximum visibility for your research: over $100 \mathrm{M}$ website views per year

At $\mathrm{BMC}$, research is always in progress.

Learn more biomedcentral.com/submissions 